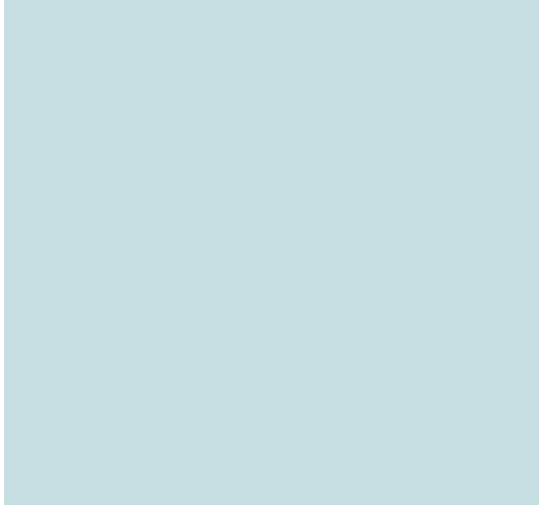

\title{
Seminal Vesicle Invasion in
} Prostate Cancer: Evaluation by Using Multiparametric Endorectal MR Imaging ${ }^{1}$

Fatma Nur Soylu, MD

Yahui Peng, PhD

Yulei Jiang, PhD

Shiyang Wang, PhD

Christine Schmid-Tannwald, MD

Ila Sethi, MD

Scott Eggener, MD

Tatjana Antic, MD

Aytekin Oto, MD
Purpose:

Materials and Methods:

Results:

${ }^{1}$ From the Department of Radiology (F.N.S., Y.P., Y.J., S.W., C.S., I.S., A.O.), Department of Surgery, Section of Urology (S.E.), and Department of Pathology (T.A.), University of Chicago, 5841 S Maryland Ave, MC 2026, Chicago, IL 60637. From the 2012 RSNA Annual Meeting. Received July 3, 2012; revision requested August 20; revision received September 27; accepted 0ctober 30; final version accepted December 13. Supported in part by the U.S. Army Medical Research and Materiel Command Prostate Cancer Research Program through an Idea Development Award PC093485. Address correspondence to A.O. (e-mail: aoto@radiology.bsd.uchicago.edu).

o RSNA, 2013
To retrospectively evaluate the diagnostic performance of multiparametric endorectal magnetic resonance (MR) imaging, including T2-weighted, diffusion-weighted (DW), and dynamic contrast material-enhanced (DCE) MR techniques, for the diagnosis of seminal vesicle invasion (SVI) and to determine the incremental value of DW MR and DCE MR images.

This retrospective HIPAA-compliant study was approved by the institutional review board, with a waiver of informed consent. The study included 131 patients (mean age, 68 years; range, 43-75 years) who underwent endorectal MR imaging before radical prostatectomy between January 2007 and April 2010. Two radiologists (A: experienced, B: less experienced) estimated the likelihood of SVI by using a five-point ordinal scale in three image-viewing settings: T2weighted images alone; T2-weighted and DW MR images; and T2-weighted, DW MR, and DCE MR images. Sensitivity, specificity, positive predictive value, negative predictive value, and area under the receiver operating characteristic curve (AUC) were calculated. Confidence intervals estimated with bootstrapping and the McNemar test or Fisher exact test were used to compare sensitivity, specificity, positive predictive value, and negative predictive value.

Of the 131 patients, 23 (17.6\%) had SVI identified after surgery. Review of T2-weighted MR images alone resulted in high specificity (93.1\% and $93.6 \%$, for radiologists $\mathrm{A}$ and $\mathrm{B}$, respectively) and high negative predictive value (94.8\% and $94.0 \%)$ but moderate sensitivity (59\% and $52 \%)$ and positive predictive value (52\% and $50 \%)$. Review of T2-weighted and DW MR images significantly improved specificity $(96.6 \%[P=.02]$ and $98.3 \%[P=.003])$ and positive predictive value $(70 \%[P<.05]$ and $79 \%[P$ $<.05])$ without significantly improving AUC. Additional review of DCE MR images did not yield further incremental improvement.

Conclusion: Additional review of DW MR images improves specificity and positive predictive value in SVI detection compared with reviewing T2-weighted images alone. Addition of DCE MR images to this combination, however, does not provide incremental value for diagnosis of SVI.

${ }^{\circ}$ RSNA, 2013

Supplemental material: http://radiology.rsna.org/lookup /suppl/doi:10.1148/radiol.13121319/-/DC1 
S eminal vesicle (SV) invasion (SVI) is associated with increased risk of lymph node metastasis and tumor recurrence in patients with prostate cancer, and therefore knowledge of its presence at the time of diagnosis is an important factor for prognosis assessment and patient treatment (1-6). The emergence of focal therapy options has further increased the importance of accurate detection because focal therapy is not suitable for treating locally aggressive cancers, including those that have invaded the SVs. Despite downward stage migration of prostate cancer since the advent of serum prostate-specific antigen testing, the prevalence of SVI has remained around $5 \%-23 \%$ in surgical series (1,5,7-19). Presurgical clinical

\section{Advances in Knowledge}

- The accuracy in the diagnosis of seminal vesicle invasion (SVI) as characterized by the area under the receiver operating characteristic curve and compared with multiple-reader multiple-case receiver operating characteristic analysis is similar $(P=.55)$ between reviewing multiparametric MR images and reviewing T2-weighted MR images alone (0.89 \pm 0.05 [standard deviation] vs $0.89 \pm 0.04$ for an experienced radiologist; $0.84 \pm 0.04$ vs $0.79 \pm 0.04$ for a less experienced radiologist, respectively).

- Reviewing diffusion-weighed (DW) MR images in addition to T2-weighted MR images helps improve specificity (from $93.1 \%$ to $96.6 \%[P=.02]$ for an experienced radiologist; from $93.6 \%$ to 98.3\% $[P=.003]$ for a less experienced radiologist) and positive predictive value (from $52 \%$ to $70 \%[P<.05]$ for an experienced radiologist; from $50 \%$ to $79 \%[P<.05]$ for a less experienced radiologist).

- Dynamic contrast-enhanced MR images provide no further incremental value to T2-weighted and DW MR images for the diagnosis of SVI. variables, such as serum prostate-specific antigen level, biopsy Gleason score, and presence of tumor at the prostate base at biopsy, commonly are used to assess the risk of SVI and to counsel patients on treatment selection $(3,8)$. However, these parameters do not provide anatomic information and cannot accurately predict the presence or location of SVI (20).

Endorectal magnetic resonance (MR) imaging has demonstrated promising results in the detection of SVI, showing high specificity $(81 \%-100 \%)$ but varying sensitivity (23\%-93\%) (20-26). These studies have used various imaging techniques or their combinations: T2-weighted imaging alone, T2-weighted and diffusion weighted (DW) MR imaging, T2-weighted and dynamic contrast material-enhanced (DCE) MR imaging, and so forth. Recently, a consensus has emerged on the use of multiparametric MR imaging protocols composed of T2-weighted, DW $\mathrm{MR}$, and DCE MR imaging sequences for detection and local staging of prostate cancer (27). Thus, the purpose of this study was to retrospectively evaluate the diagnostic performance of multiparametric endorectal MR imaging, including T2weighted, DW MR, and DCE MR techniques, in the diagnosis of SVI and the incremental diagnostic value of DW MR and DCE MR images.

\section{Materials and Methods}

\section{Patients}

This retrospective study was compliant with the Health Insurance Portability and Accountability Act and was approved by our institutional review board, with a waiver for informed patient consent. This study was partially supported by the Department of Defense Idea Development Grant (award

\section{Implication for Patient Care}

- Multiparametric MR imaging improves specificity and positive predictive value in the diagnosis of SVI, compared with T2-weighted imaging alone, but does not improve sensitivity. number W81XWH-10-1-0570). We searched clinical records at our institution to identify consecutive patients who underwent endorectal multiparametric MR imaging between January 2007 and April 2010 and subsequently underwent radical prostatectomy. We excluded patients with previous history of radiation, chemotherapy, or hormonal therapy for treatment of prostate cancer $(n=3)$ or incomplete MR examination (ie, image distortion or artifacts caused by the endorectal coil or patient incompliance with intravenous injection of contrast agent) $(n=5)$. The study included 131 patients whose median age was 68 years (age range, 43-75 years). Median prostate-specific antigen level was $12.1 \mathrm{ng} / \mathrm{mL}(12.1 \mu \mathrm{g} / \mathrm{L})$ (range, 1.5-65.0 ng/mL [1.5-65.0 $\mu \mathrm{g} / \mathrm{L}])$, and median Gleason score was 7 (range, 6-9). The average time between MR examination and prostate resection was 68.8 days (range, $6-427$ days).

\section{MR Techniques and Image Acquisition}

All MR imaging examinations were performed by using an endorectal coil (Medrad, Warrendale, Pa) and a phased-array surface coil, with 1.5-T MR imagers (Excite HD, GE Healthcare, Waukesha, Wis; Achieva, Philips Healthcare, Eindhoven, the Netherlands). Seventy-three

\section{Published online before print}

10.1148/radiol.13121319 Content code: GU

Radiology 2013; 267:797-806

\section{Abbreviations:}

$A D C=$ apparent diffusion coefficient

$A U C=$ area under the receiver operating characteristic curve

DCE = dynamic contrast material enhanced

$\mathrm{DW}=$ diffusion weighted

$\mathrm{SV}=$ seminal vesicle

SVI $=$ SV invasion

\section{Author contributions:}

Guarantors of integrity of entire study, F.N.S., A.0.; study concepts/study design or data acquisition or data analysis/ interpretation, all authors; manuscript drafting or manuscript revision for important intellectual content, all authors; manuscript final version approval, all authors; literature research, F.N.S., C.S., I.S., S.E., A.O.; clinical studies, F.N.S., S.W., A.O.; statistical analysis, Y.P., Y.J., A.O.; and manuscript editing, F.N.S., Y.P., Y.J., S.E., T.A., A.O.

Conflicts of interest are listed at the end of this article. 
patients were imaged with the GE Healthcare imager, and 58 patients were imaged with the Philips Healthcare imager. Immediately before MR image acquisition, $1 \mathrm{mg}$ glucagon (Lilly, Indianapolis, Ind) was injected intramuscularly to suppress peristalsis. The entire prostate was imaged. A parallel imaging factor of two was used in all sequences. The following imaging series were obtained: axial, coronal, and sagittal T2-weighted fast spin-echo images (section thickness, $3 \mathrm{~mm}$ ); axial T1-weighted fast spin-echo images; axial free-breathing DW MR images ( $b=0$ and 1000 or $1500 \mathrm{sec} / \mathrm{mm}^{2}$ ); and axial free-breathing DCE MR images. Acquisition of T1-weighted DCE MR images of the entire prostate started approximately 30 seconds before intravenous administration of $0.1 \mathrm{mmol}$ gadodiamide (Omniscan; GE Healthcare, Princeton, NJ) per kilogram of body weight and a $20-\mathrm{mL}$ saline flush at a rate of $2.0 \mathrm{~mL} / \mathrm{sec}$. Detailed image acquisition protocols are given in Tables 1 and 2 and in Appendix E1 (online).

\section{MR Image Analysis}

MR images were retrospectively and independently reviewed by two radiologists (A.O., radiologist A, 9 years of prostate MR imaging experience; and F.N.S., radiologist B, 2 years of prostate MR imaging experience). The radiologists were blinded to clinical, surgical, and histologic findings except for the knowledge that each patient had biopsy-proved prostate cancer.

Apparent diffusion coefficient (ADC) maps were generated from DW MR images by using commercial diffusion analysis software (Advantage Windows, version 4.2.3, GE Healthcare, Milwaukee, Wis; ViewForum, Philips Healthcare). DCE MR images were processed on a commercial workstation (DynaCAD for Prostate; Invivo, Gainesville, Fla). For each sequential time point, the DCE MR images were subtracted from the corresponding precontrast images, and both DCE MR and the subtracted images were reviewed by the radiologists.

The two radiologists reviewed the MR images in three image-viewing set-

\begin{tabular}{|c|c|c|c|}
\hline \multicolumn{4}{|c|}{ Imaging Parameters for Prostate Examination with 1.5-T GE Healthcare MR Imager } \\
\hline Parameter & T2-weighted MR Imaging & DW MR Imaging & DCE MR Imaging \\
\hline $\begin{array}{l}\text { Repetition time (msec)/echo } \\
\text { time (msec) }\end{array}$ & $3200-3500 / 90-100$ & $7000-8000 / 80-90$ & $3.5-3.9 / 1.6-1.9$ \\
\hline Matrix & $192 \times 256$ & $128 \times 128-224$ & $160 \times 256$ \\
\hline Flip angle (degrees) & 90 & 90 & 10 \\
\hline Section thickness (mm) & 3 & 4 & $3-4$ \\
\hline Field of view $(\mathrm{cm})$ & $14-16$ & $14-18$ & $14-18$ \\
\hline No. of signals acquired & 4 & 4 & NA \\
\hline$b$ Value $\left(\mathrm{sec} / \mathrm{mm}^{2}\right)$ & NA & $0,1000,1500$ & NA \\
\hline Temporal resolution (sec) & NA & NA & $5-12$ \\
\hline
\end{tabular}

Note.-An array spatial sensitivity encoding technique (parallel imaging) factor of two was applied to all of the sequences $\mathrm{NA}=$ not applicable.

\begin{tabular}{|c|c|c|c|}
\hline \multicolumn{4}{|c|}{$\begin{array}{l}\text { Imaging Parameters for Prostate Examination with 1.5-T Philips Healthcare MR } \\
\text { Imager }\end{array}$} \\
\hline Parameter & T2-weighted MR Imaging & DW MR Imaging & DCE MR Imaging \\
\hline $\begin{array}{l}\text { Repetition time (msec)/echo } \\
\text { time (msec) }\end{array}$ & $4300-5000 / 120$ & $3800-4200 / 80-90$ & $5.5 / 2.1$ \\
\hline Matrix & $204 \times 256$ & $128 \times 128$ & $199 \times 292$ \\
\hline Flip angle (degrees) & 90 & 90 & 10 \\
\hline Section thickness $(\mathrm{mm})$ & 3 & 4 & $3-4$ \\
\hline Field of view $(\mathrm{cm})$ & $14-18$ & $14-18$ & $14-18$ \\
\hline No. of signals acquired & 4 & 4 & NA \\
\hline$b$ Value $\left(\mathrm{sec} / \mathrm{mm}^{2}\right)$ & NA & $0,1000,1500$ & NA \\
\hline Temporal resolution (sec) & NA & NA & $3-5$ \\
\hline
\end{tabular}

Note. - An effective sensitivity encoding (parallel imaging) factor of two was applied to all of the sequences. NA = not applicable

tings, consecutively, during the same reading session. First, they evaluated T2-weighted MR images (axial, coronal, and sagittal views), and precontrast T1weighted images were also evaluated to rule out false-positive findings caused by postbiopsy hemorrhage (28). Then, they reviewed ADC maps in conjunction with T2-weighted images. Finally, they evaluated DCE MR images together with T2-weighted images and ADC maps. The order of the image review was the same for each patient, and the radiologists were aware of their interpretation of the T2-weighted images when viewing the ADC maps and DCE MR images. In cases with restricted diffusion in the SVs (dark signal on ADC map), DW MR images were reviewed to confirm corresponding increased signal intensity at the same location to exclude the possibility of inherently dark T2 signal leading to a false-positive ADC map indication for restricted diffusion.

\section{Diagnostic Criteria and Image Interpretation}

MR criteria for SVI diagnosis were based on criteria previously reported in the literature $(22,24,29,30)$. The criteria for SVI diagnosis on T2 weighted images and ADC maps were at least one of the following: disruption or loss of normal architecture of the SV, focal or diffuse areas of low signal intensity within the SV (without corresponding high signal intensity on T1-weighted images at the same location), asymmetric thickening or 


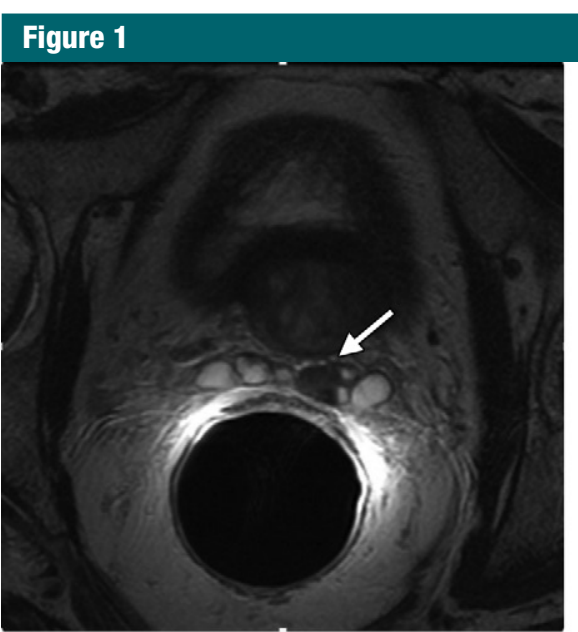

a.

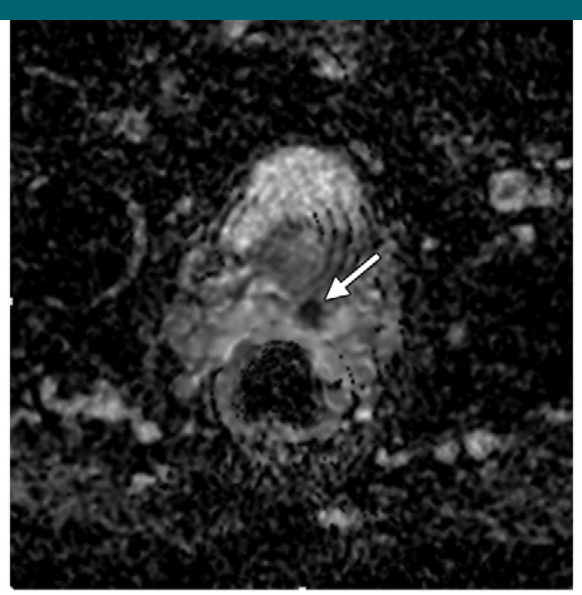

b.

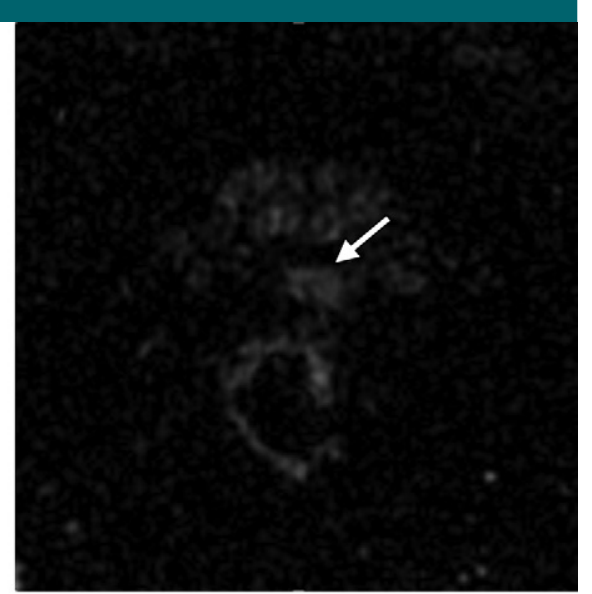

c.

Figure 1: Left SVI of histopathologically proved prostate cancer in 71-year-old man with a prostate-specific antigen level of 15 ng/mL (15 $\mu$ g/L) and a Gleason score of $9(4+5)$. (a) Transverse T2-weighted fast spin-echo (4200/80) MR image shows a focal, homogeneously low-signal-intensity lesion in the left SV (arrow) representing a high possibility of SVI. (b) ADC map (2999/65) shows the focal low-signal-intensity lesion in left SV corresponding to a (arrow). (c) Subtracted DCE MR image (precontrast image was subtracted from DCE image at the same level) corresponding to a and $\mathbf{b}$ shows the early and intense enhancement in the left SV lesion (arrow). Both readers correctly scored this lesion as 5 (definite SVI) on the basis of T2-weighted image, ADC map, and DCE MR subtraction image.

irregularity of the SV wall, and evident tumor at the prostate base extending to the SV. On DCE MR images, criteria for SVI diagnosis were at least one of the following: focal areas of enhancement within the $\mathrm{SV}$, asymmetric or irregular SV wall thickening or enhancement, and evident tumor at the base of the prostate extending to the SV. On the basis of these criteria, each radiologist subjectively estimated the likelihood of SVI for each of the three image-viewing settings (T2-weighted images only; T2-weighted and DW MR images; and T2-weighted, DW MR, and DCE MR images) by using a five-point ordinal scale: 1, SVI not present; 2, SVI probably not present but cannot be ruled out (no clear evidence); 3, SVI possibly present (suspicious); 4, SVI probably present (highly suspicious); and 5, SVI definitely present (Fig 1) (20).

Diagnostic performance was analyzed per SV and per patient. For the per-SV analysis, each SV was treated as a single unit for statistical analysis (thus, the 131 patients had $262 \mathrm{SVs}$ ). For the per-patient analysis, each patient was treated as a single unit for analysis, and each radiologist's ratings of the left and right SVs were combined by retaining the score most indicative of SVI (eg, if the left SV were given a score of 2 and the right SV a score of 3 , then the score for the patient was 3 ).

\section{Histopathologic Analysis}

Radical prostatectomy specimens of the entire prostate were fixed in 5\% buffered formalin for 24 hours. SVs were separated from the prostate and processed separately. After dehydration, the specimens were cut serially into 4-mm-thick blocks from apex to base in transverse planes. Each block was then halved or quartered, depending on its size; microtomed into 7-8- $\mu \mathrm{m}$ slices; and stained with hematoxylin-eosin. The hematoxylin-eosin-stained sections of the 131 patients with prostate cancer were reviewed by a genitourinary pathologist (T.A.). SVI was defined microscopically as prostate cancer cell invasion into the wall of the SV. SVI presence and location (left vs right side) were recorded for each patient.

\section{Statistical Analysis}

Serum prostate-specific antigen levels in patients with and those without SVI were compared by using a two-sample $t$ test. Sensitivity, specificity, positive predictive value, and negative predictive value in the diagnosis of SVI were calculated. True-positive diagnosis was defined as a histopathologically confirmed SVI that was rated by a radiologist as 3 or greater according to the five-point scale, and true-negative diagnosis was defined as histopathologically confirmed lack of SVI that was rated by a radiologist as 2 or less according to the same scale.

Confidence intervals in the estimated sensitivity, specificity, positive predictive value, and negative predictive value, as well as in the estimated differences in these performance parameters between image-viewing settings, were estimated from 5000 bootstrapping samples (31). Bootstrapping samples were generated by sampling cases (with replacement) and estimating all performance parameters simultaneously from the sampled cases. For each parameter of interest, 95\% confidence interval was estimated by eliminating the top and bottom $2.5 \%$ of all bootstrapping estimates of that parameter. In addition, when 95\% confidence intervals indicated statistically significant differences between imageviewing settings, the binomial exact version of the McNemar test or the Fisher exact test (32) was also applied.

Maximum-likelihood proper binormal receiver operating characteristic 


\section{Table 3}

\section{Per-SV Diagnostic Performance Indexes for Detecting SVI on Endorectal Multiparametric MR Images}

\begin{tabular}{|c|c|c|c|c|c|}
\hline Parameter & T2-weighted MR Imaging & $\begin{array}{l}\text { Incremental } \\
\text { Difference (\%) }\end{array}$ & $\begin{array}{l}\text { T2-weighted and } \\
\text { DW MR Imaging }\end{array}$ & $\begin{array}{l}\text { Incremental } \\
\text { Difference (\%) }\end{array}$ & $\begin{array}{l}\text { T2-weighted, DW, and } \\
\text { DCE MR Imaging }\end{array}$ \\
\hline \multicolumn{6}{|l|}{ Sensitivity } \\
\hline Radiologist A & $59(17 / 29)[40.0,76.5]$ & $-6.2,21.2$ & $66(19 / 29)[48.0,82.8]$ & $0.0,0.0$ & $66(19 / 29)[48.0,82.8]$ \\
\hline Radiologist B & $52(15 / 29)[33.3,70.0]$ & $-17.2,16.7$ & $52(15 / 29)[33.3,70.3]$ & $-10.0,10.3$ & $52(15 / 29)[33.3,70.0]$ \\
\hline \multicolumn{6}{|l|}{ Specificity } \\
\hline Radiologist A & $93.1(217 / 233)[89.7,96.2]$ & $0.9,6.2$ & $96.6(225 / 233)[94.1,98.7]$ & $-1.3,1.3$ & $96.6(225 / 233)[94.1,98.7]$ \\
\hline Radiologist B & $93.6(218 / 233)[90.2,96.6]$ & $1.8,7.9$ & $98.3(229 / 233)[96.5,99.6]$ & $0.0,1.3$ & $98.7(230 / 233)[97.0,100.0]$ \\
\hline \multicolumn{6}{|c|}{ Positive predictive value } \\
\hline Radiologist A & $52[17 / 33][33.3,69.0]$ & $6.6,33.3$ & $70(19 / 27)[52.0,87.5]$ & $-7.6,7.5$ & $70(19 / 27)[52.0,87.0]$ \\
\hline Radiologist B & $50(15 / 30)[31.6,67.9]$ & $10.8,48.8$ & $79(15 / 19)[58.8,95.2]$ & $-2.0,15.4$ & $83(15 / 18)[63.6,100.0]$ \\
\hline \multicolumn{6}{|c|}{ Negative predictive value } \\
\hline Radiologist A & $94.8(217 / 229)[91.8,97.4]$ & $-0.6,2.7$ & $95.7(225 / 235)[93.1,98.3]$ & $-0.1,0.1$ & $95.7(225 / 235)[93.1,98.3]$ \\
\hline Radiologist B & $94.0(218 / 232)[90.8,96.9]$ & $-1.7,2.2$ & $94.2(229 / 243)[91.2,97.1]$ & $-1.1,1.2$ & $94.3(230 / 244)[91.3,97.1]$ \\
\hline
\end{tabular}

Note.-Data are performance indexes as percentages, with numbers of SVs in parentheses and $95 \%$ confidence intervals as percentages in brackets.

\section{Table 4}

\begin{tabular}{|c|c|c|c|c|c|}
\hline Parameter & T2-weighted MR Imaging & $\begin{array}{l}\text { Incremental } \\
\text { Difference (\%) }\end{array}$ & $\begin{array}{l}\text { T2-weighted and } \\
\text { DW MR Imaging }\end{array}$ & $\begin{array}{l}\text { Incremental } \\
\text { Difference (\%) }\end{array}$ & $\begin{array}{l}\text { T2-weighted, DW, and } \\
\text { DCE MR Imaging }\end{array}$ \\
\hline \multicolumn{6}{|l|}{ Sensitivity } \\
\hline Radiologist A & $65(15 / 23)[45.5,84.6]$ & $0.0,28.6$ & $78(18 / 23)[60.0,94.4]$ & $0.0,0.0$ & $78(18 / 23)[60.0,94.4]$ \\
\hline Radiologist B & $56.5(13 / 23)[35.3,76.9]$ & $-15.0,23.8$ & $61(14 / 23)[40.0,80.8]$ & $-12.0,12.5$ & $61(14 / 23)[40.0,80.0]$ \\
\hline \multicolumn{6}{|l|}{ Specificity } \\
\hline Radiologist A & $90.7(98 / 108)[84.8,96.0]$ & $0.9,10.8$ & $96.3(104 / 108)[92.3,99.1]$ & $-2.8,2.8$ & $96.3(104 / 108)[92.4,99.1]$ \\
\hline Radiologist B & $88.9(96 / 108)[82.7,94.3]$ & $2.8,14.2$ & $97.2(105 / 108)[93.6,100.0]$ & $0.0,2.9$ & $98.2(106 / 108)[95.2,100.0]$ \\
\hline \multicolumn{6}{|c|}{ Positive predictive value } \\
\hline Radiologist A & $60[15 / 25][40.0,79.3]$ & $5.6,39.5$ & $82(18 / 22)[64.0,95.8]$ & $-10.7,11.5$ & $82(18 / 22)[64.7,95.8]$ \\
\hline Radiologist B & $52(13 / 25)[32.0,71.4]$ & $11.2,51.1$ & $82(14 / 17)[61.5,100.0]$ & $-1.9,18.2$ & $88(14 / 16)[68.8,100.0]$ \\
\hline \multicolumn{6}{|c|}{ Negative predictive value } \\
\hline Radiologist A & $92.5(98 / 106)[87.4,97.1]$ & $0.3,6.3$ & $95.4(104 / 109)[91.2,99.1]$ & $-0.1,0.1$ & $95.4(104 / 109)[91.2,99.1]$ \\
\hline Radiologist B & $90.6(96 / 106)[84.8,95.4]$ & $-2.1,5.4$ & $92.1(105 / 114)[87.0,96.5]$ & $-2.3,2.5$ & $92.2(106 / 115)[87.1,96.6]$ \\
\hline
\end{tabular}

Note.-Data are performance indexes as percentages, with numbers of SVs in parentheses and 95\% confidence intervals as percentages in brackets.

curves were estimated to evaluate the diagnostic performance of the two radiologists in the three image-viewing settings, and the area under the receiver operating characteristic curve (AUC) was used as a summary performance index (33). Multiple-reader multiple-case analysis with jackknifing and analysis of variance was used to compare receiver operating characteristic curves (34). Agreement between the two radiologists was analyzed with $\kappa$, with the following interpretation of the $\kappa$ value: $0.00-0.20$, poor agreement; 0.21-0.40, fair agreement; 0.41-0.60, moderate agreement; $0.61-0.80$, substantial agreement; and 0.81-1.00, almost perfect agreement (35).

All $P$ values were two-sided, and $P$ less than .05 was considered to indicate a statistically significant difference. Statistical significance was also considered demonstrated if the $95 \%$ confidence intervals did not include zero.

\section{Results}

\section{Histopathologic Findings}

Histopathologic analysis identified 29 (11.1\% [29 of 262]) SVs positive for
SVI in 23 (17.6\% [23 of 131]) patients: six patients had bilateral, eight had left-side only, and nine had right-side only SVI. The median baseline serum prostate-specific antigen level was 13.3 $\mathrm{ng} / \mathrm{mL}(13.3 \mu \mathrm{g} / \mathrm{L}$ ) (range, 4.5-37.0 ng/ $\mathrm{mL}[4.5-37.0 \mu \mathrm{g} / \mathrm{L}])$ for the men with SVI and $8.8 \mathrm{ng} / \mathrm{mL}(8.8 \mu \mathrm{g} / \mathrm{L})$ (range, $1.5-65.0 \mathrm{ng} / \mathrm{mL}[1.5-65.0 \mu \mathrm{g} / \mathrm{L}])$ for the men without SVI; their differences were statistically significant $(P=.002)$.

\section{Multiparametric MR Imaging Findings}

Table 3 (per SV) and Table 4 (per patient) summarize sensitivity, specificity, positive 


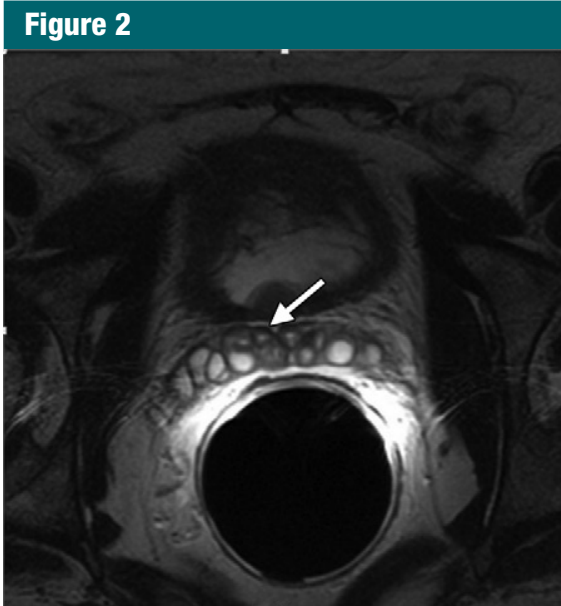

a.

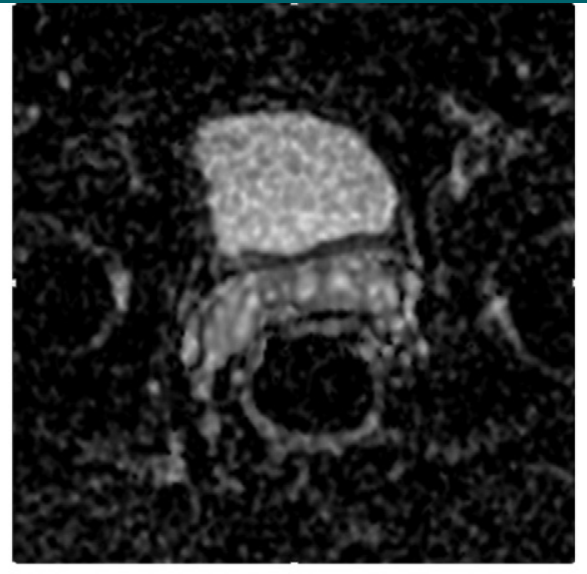

b.

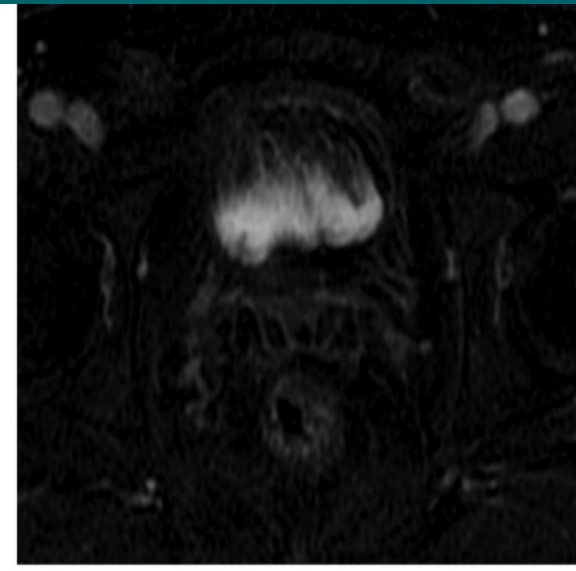

c.

Figure 2: Images in 55-year-old man with prostate-specific antigen level of $12 \mathrm{ng} / \mathrm{mL}(12 \mu \mathrm{g} / \mathrm{L})$, a Gleason score of 8 (4 + 4), and findings suggestive of SV involvement on T2-weighted images. (a) Transverse T2-weighted fast spin-echo (4200/80) MR image shows a suspicious wall thickening, loss of normal architecture, and focal low signal intensity in right SV (arrow) representing a possibility of SVI. (b) ADC map (2999/65) and (c) DCE MR subtraction image corresponding to a show no evidence of focal dark spot or enhancement, respectively, within the lumen of the right SV that suggests invasion. Both readers correctly decreased the score as 3, 2, and 2 ( 3 = possible SVI, 2 = probably no SVI) on the basis of T2-weighted image, ADC map, and subtraction DCE MR image, respectively. Histopathologic examination findings showed no SVI.

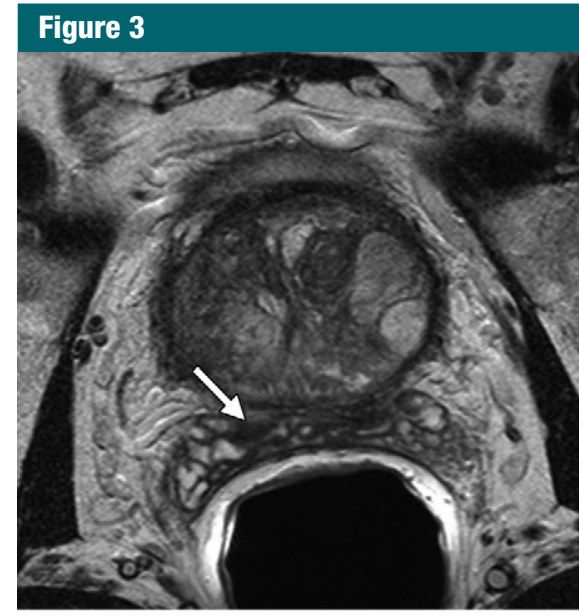

a.

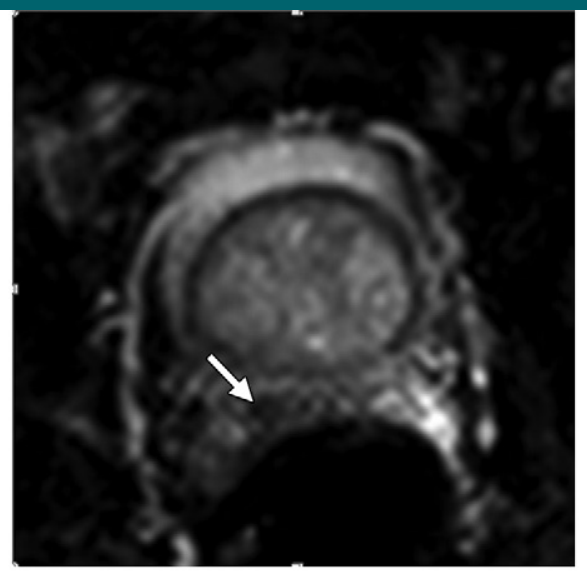

b.

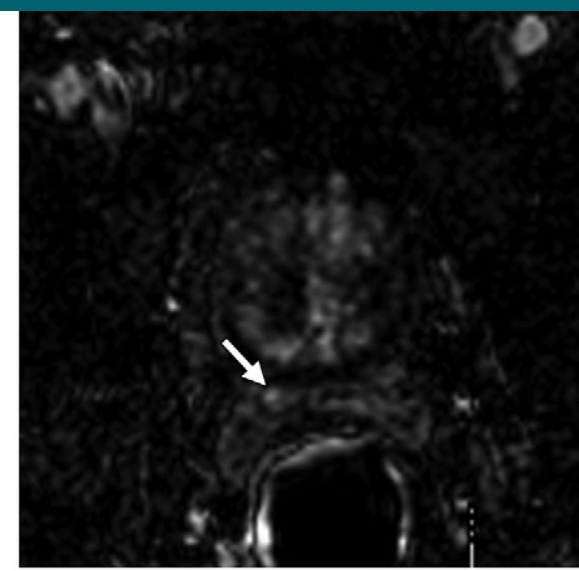

c.

Figure 3: Images in 65-year-old man with a prostate-specific antigen level of $20.2 \mathrm{ng} / \mathrm{mL}(20.2 \mu \mathrm{g} / \mathrm{L})$ and a Gleason score of $8(4+4)$ with right SVI of prostate cancer. (a) Transverse T2-weighted fast spin-echo (4200/80) MR image shows equivocal wall thickening in the right SV (arrow) representing a possibility of SVI. (b) ADC map (2999/65) shows wall thickening and focal low signal intensity in right SV (arrow) corresponding to a. (c) Subtraction DCE MR image corresponding to a and $\mathbf{b}$ shows the early and focal enhancement in the wall of right SV (arrow). The experienced reader increased the score correctly as 2,4 , and 4 for this lesion ( $2=$ probably no SVI, 4 = probable SVI) on the basis of T2-weighted image, ADC map, and subtraction DCE MR image, respectively, when the less experienced reader could not detect the lesion. This lesion was proved to be SVI at histopathologic examination.

predictive value, and negative predictive value and their differences between the three image-viewing settings. In the per$\mathrm{SV}$ analysis, when reviewing T2-weighted images alone, both radiologists achieved high specificity $(93.1 \%$ and $93.6 \%$, for radiologists $\mathrm{A}$ and $\mathrm{B}$, respectively) and high negative predictive value $\mathbf{9 4 . 8 \%}$ and $94.0 \%)$ but moderate sensitivity (59\% and $52 \%$ ) and positive predictive value (52\% and 50\%) (Fig 1). The addition of DW MR images for diagnostic image review significantly improved specificity and positive predictive value (specificity: from $93.1 \%$ to $96.6 \%[P=.02]$ and from $93.6 \%$ to $98.3 \%[P=.003]$ for radiologists $\mathrm{A}$ and $\mathrm{B}$, respectively, binomial test) (positive predictive value: from 52\% to $70 \%[P<.05]$ and from $50 \%$ to $79 \%[P$ $<.05]$ for radiologists $\mathrm{A}$ and $\mathrm{B}$, respectively) (Figs 2, 3). Table 3 shows 95\% 


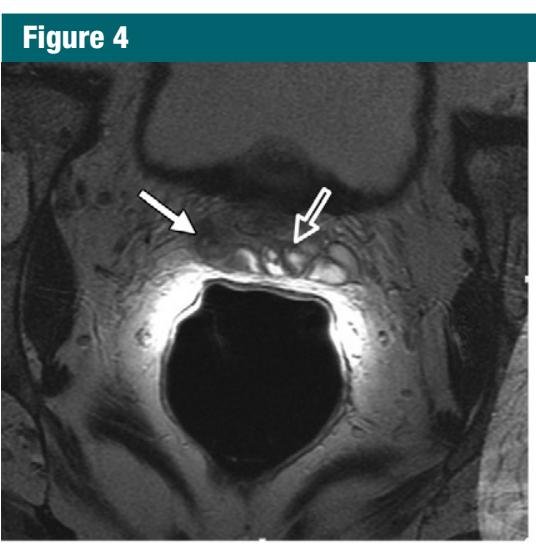

a.

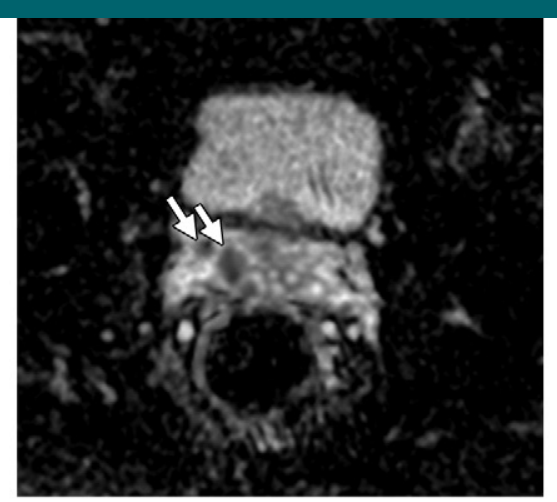

b.

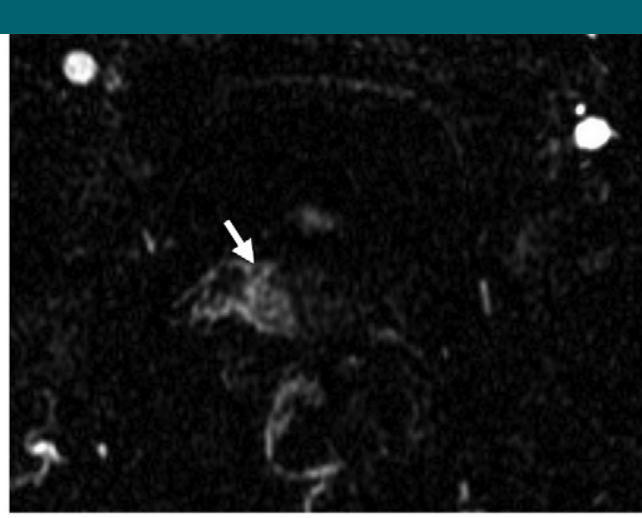

c.

Figure 4: Bilateral SVI of prostate cancer correctly identified on the right side, but missed on the left side in 50-year-old man, with prostate-specific antigen level of $28 \mathrm{ng} / \mathrm{mL}(28 \mu \mathrm{g} / \mathrm{L})$ and a Gleason score of $8(4+4)$. (a) Transverse T2-weighted fast spin-echo (4200/80) MR image shows diffuse low signal intensity with loss of normal architecture in right SV (solid arrow) representing a high possibility of SVI and a suspect wall thickening on left SV (open arrow). (b) ADC map (2999/65) corresponding to a also shows focal areas of low signal intensity (arrows) with loss of normal architecture in right SV but no signs of SVI on the left side. (c) Subtraction DCE MR image corresponding to $\mathbf{a}$ and $\mathbf{b}$ shows diffuse early enhancement on right SV (arrow) but no findings of SVI on the left side. Both readers correctly scored the right SV lesion as 5 (definite SVI) on both images. For left SV, both readers decreased the score as 3, 2, and 2 and 2, 1, and 1 for experienced and less experienced readers, respectively ( $3=$ possible SVI, 2 = probably no SVI, 1 = no SVI) on consecutive T2-weighted image, ADC map, and subtraction image. From the histopathologic findings, bilateral SVI was proved.

\section{Table 5}

\begin{tabular}{|c|c|c|c|}
\hline \multicolumn{4}{|c|}{ Per-Patient AUCs for Detecting SVI on Endorectal Multiparametric MR Images } \\
\hline Parameter & T2-weighted MR Imaging & $\begin{array}{l}\text { T2-weighted and } \\
\text { DW MR Imaging }\end{array}$ & $\begin{array}{l}\text { T2-weighted, DW, and } \\
\text { DCE MR Imaging }\end{array}$ \\
\hline \multicolumn{4}{|c|}{ Analysis of radiologist $\mathrm{A}$} \\
\hline Per SV & $0.89 \pm 0.04$ & $0.88 \pm 0.05$ & $0.89 \pm 0.05$ \\
\hline Per patient & $0.90 \pm 0.04$ & $0.89 \pm 0.05$ & $0.92 \pm 0.04$ \\
\hline \multicolumn{4}{|c|}{ Analysis of radiologist $B$} \\
\hline Per SV & $0.79 \pm 0.04$ & $0.83 \pm 0.04$ & $0.84 \pm 0.03$ \\
\hline Per patient & $0.79 \pm 0.05$ & $0.85 \pm 0.04$ & $0.86 \pm 0.04$ \\
\hline
\end{tabular}

Note.-Data are means \pm standard deviations.

confidence intervals. Furthermore, Table 3 shows improvements in sensitivity and negative predictive value, which were not statistically significant. The addition of DCE MR images for diagnostic image review did not produce further statistically significant improvement (Fig 4). The per-patient analysis results were similar to the per-SV analysis results in terms of sensitivity, specificity, positive predictive value, and negative predictive value (Table 4 ). The only notable difference was that for the experienced reader (radiologist A), negative predictive value also improved significantly (from 92.5\% to $95.4 \%[P<.05])$ when DW MR images were read together with T2-weighted images.

The per-SV-analysis AUCs for diagnosis of SVI were $0.89 \pm 0.04$ (standard deviation), $0.88 \pm 0.05$, and 0.89 \pm 0.05 for reviewing $\mathrm{T} 2$-weighted, T2weighted and DW MR, and T2-weighted, DW MR, and DCE MR images by radiologist $\mathrm{A}$ and $0.79 \pm 0.04,0.83 \pm$ 0.04 , and $0.84 \pm 0.04$ by radiologist B, respectively (Table 5). Per-patient results were similar (Table 5 ). The addition of DW MR and DCE MR images to the review of $\mathrm{T} 2$-weighted images slightly improved the performance of the less experienced radiologist (radiologist B), but multiple-reader multiplecase receiver operating characteristic analysis showed no statistically significant difference between the AUCs either between the two radiologists or between the three image-viewing settings $(P=.55$, per-SV analysis; $P=.18$, per-patient analysis). These results in sensitivity, specificity, positive predictive value, negative predictive value, and AUC were largely unchanged when each patient was treated as a unit for statistical analysis, by combining the analysis of the left and right SVs with the single assessment that is most indicating of possible SVI (eg, if the left SV were assessed as 2 and the right SV assessed as 3 , then the patient would be assessed as 3 ). These results are not shown for brievity.

The per-SV analysis $\kappa$ statistics characterizing agreement between the radiologists were $0.66,0.67$, and 0.69 for reviewing $\mathrm{T} 2$-weighted; $\mathrm{T} 2$-weighted and DW; and T2-weighted, DW, and DCE MR images; the per-patient analysis $\kappa$ statistics were $0.65,0.67$, and 0.69 , respectively. Thus, interobserver agreement was substantial (35). 


\section{Discussion}

Our results show that, in both the per-SV and per-patient analyses, review of DW MR images in addition to T2-weighted images helped both radiologists to improve specificity and positive predictive value for the diagnosis of SVI. There was also some indication of improved sensitivity and negative predictive value in the perSV analysis, which fell short of being statistically significant, while negative predictive value demonstrated significant improvement for the experienced reader in the per-patient analysis. However, adding DCE MR images showed no incremental value to the combination of T2-weighted and DW MR images.

The use of MR imaging for prostate cancer local staging remains controversial (27). Wang et al (20) reported an AUC of 0.76 for the diagnosis of SVI by using T2-weighted MR images, which is greater than that of several other clinical variables $(\mathrm{AUC}=0.62-0.73$ for prostate-specific antigen level, Gleason grade, and clinical stage, etc) and concluded that endorectal MR imaging produces significant incremental value to the Kattan nomogram for predicting SVI. However, other studies have reported varying sensitivity (23\%-80\%) and specificity $(81 \%-99 \%)$ based on review of $\mathrm{T} 2$-weighted images alone $(9-12,22,29,36)$. Low signal intensity within the SV, loss of normal SV architecture, and presence of tumor at the prostate base were reported to produce moderate sensitivity but high specificity for the diagnosis of SVI (22). By using these T2-weighted imaging features, Sala et al (22) reported sensitivity of $50 \%-79 \%$ and specificity of $97 \%-99 \%$. A meta-analysis of T2-weighted images for prostate cancer local staging also reported a receiver operating characteristic curve with high specificity $(95 \%)$ and low sensitivity (27\%) (37). Our results based on T2-weighted images (AUCs of 0.89 and 0.79 for the two radiologists reviewing $\mathrm{T} 2$-weighted images alone) were comparable to, or slightly better than, previously reported values, and we also observed high specificity $(>90 \%)$ but only moderate sensitivity $(50 \%-60 \%)$.

DW MR imaging is promising for the detection of prostate cancer and for the assessment of prostate cancer aggressiveness. However, its diagnostic performance in tumor local staging, including SVI, has not been well studied (27). Kim et al (24) reported in a series of 30 patients that combining DW MR with T2-weighted images outperformed T2-weighted images alone for SVI diagnosis by improving specificity for both an experienced radiologist and a less experienced radiologist and by improving the AUC for the less experienced radiologist. In another study, Ren et al (23) reported that adding DW MR images to T2weighted images improved the AUC for SVI diagnosis from 0.79 to 0.90 . In our study, the incremental benefit of DW MR images was improving specificity and positive predictive value, but not the AUC, for both an experienced and a less experienced radiologist. Although improvement in specificity from the addition of DW MR images to T2-weighted images was statistically significant, the absolute improvement for both readers was modest, limiting clinical importance of the improvement. In some cases, we found that ADC maps helped delineation of normal SV architecture and wall structure, which were sometimes not seen well on T2-weighted images because of a lack of SV distention or diffusely low T2 signals of the lumen.

DCE MR imaging has been reported to help improve prostate cancer staging for less experienced radiologists (25). However, only seven patients with SVI were included in that study, and review of DCE MR images helped only the less experienced radiologists detect two additional SVI cases, increasing their sensivity from $43 \%$ (three of seven) to $71 \%$ (five of seven). In our series, DCE MR images did not help in establishing the diagnosis of SVI beyond the combination of T2-weighted and DW MR images for either the experienced or less experienced radiologist.

The results of our study showed that the addition of DW MR and DCE MR images to T2-weighted images does not improve the sensitivity of SVI detection. SVI is defined histopathologically as prostate cancer tumor infiltration of the muscle wall of the SV (38). However, MR criteria for SVI detection rely heavily on luminal extension of the tumor, and microscopic invasion of the SV wall without luminal involvement or microscopic tumor invasion of the lumen is almost impossible to detect by using MR imaging, despite multiparametric imaging techniques. Furthermore, hemorrhage, focal inflammation, atrophic changes, or lack of distention also limit the diagnosis of SVI on MR images (24).

Our study had several limitations. First, our study was retrospective and had a relatively small patient sample. Second, the readers were aware of their interpretation of T2-weighted images when reviewing ADC maps and DCE MR images. Thus, this study evaluated incremental diagnostic value of DW MR and DCE MR to T2-weighted images, rather than the diagnostic value of each imaging sequence individually. Third, we did not specifically analyze the combination of DCE MR and T2weighted images alone because unlike DCE MR images, DW MR images can be acquired without the additional cost and risk of intravenous contrast agents and are therefore the first choice of additional imaging to be combined with T2-weighted images. Fourth, we studied 1.5-T MR images, and 3.0-T MR imaging has the potential to produce better diagnostic performance from increased signal-to-noise ratio and better spatial resolution. Fifth, we did not include quantitative analysis of DW MR or DCE MR images. We believe that quantitative analysis will be difficult for evaluation of SVI because of small and irregular shaped regions of interest limiting accurate measurements, at least with the current spatial resolution of these sequences. In addition, normal SVs do not demonstrate luminal enhacement. Therefore, any focal area of enhancement within the lumen of the $\mathrm{SV}$ is suspicious for tumor invasion regardless of any quantitative perfusion threshold. Future studies investigating the role of quantitative analysis with the 
use of higher resolution of DW MR and DCE MR images may be worthwhile. Sixth, $b$ values used in all cases were not the same (0 and 1000 or $1500 \mathrm{sec} /$ $\mathrm{mm}^{2}$ ). This was because of evolution of or clinical prostate MR protocol and a limitation due to the retrospective design of the study. Seventh, in the statistical analysis, each SV was treated as an independent unit; however, bilateral SVI is common, and therefore independent evaluation of each unit may be a limitation. We performed per-patient analysis to address this limitation, and the results of per-patient analysis were similar to per-SV analysis.

In summary, our results suggest that multiparametric MR imaging can accurately demonstrate SVI prior to radical prostatectomy. The combination of DW MR and T2-weighted images improves specificity and positive predictive value in the diagnosis of SVI for both experienced and relatively inexperienced radiologists, but does not improve sensitivity, in comparison with reviewing T2-weighted images alone. DCE MR images do not provide additional incremental value.

Disclosures of Conflicts of Interest: F.N.S. No relevant conflicts of interest to disclose. Y.P. No relevant conflicts of interest to disclose. Y.J. No relevant conflicts of interest to disclose. S.W. No relevant conflicts of interest to disclose. C.S. No relevant conflicts of interest to disclose. I.S. No relevant conflicts of interest to disclose. S.E. Financial activities related to the present article: none to disclose. Financial activities not related to the present article: author is consultant for Janssen; institution has grant from Myriad Genetic; author lectures for Janssen. Other relationships: none to disclose. T.A. No relevant conflicts of interest to disclose. A.O. Financial activities related to the present article: none to disclose. Financial activities not related to the present article: author is consultant for Oxford Analytica; author gives expert testimony for some law firms; author lectures for Philips Healthcare and Bracco. Other relationships: none to disclose.

\section{References}

1. Masterson TA, Pettus JA, Middleton RG, Stephenson RA. Isolated seminal vesicle invasion imparts better outcomes after radical retropubic prostatectomy for clinically localized prostate cancer: prognostic stratification of pt3b disease by nodal and margin status. Urology 2005;66(1):152-155.

2. Hull GW, Rabbani F, Abbas F, Wheeler TM, Kattan MW, Scardino PT. Cancer control with radical prostatectomy alone in 1,000 consecutive patients. J Urol 2002;167(2 Pt 1):528-534

3. Ramsden AR, Chodak G. An analysis of risk factors for biochemical progression in patients with seminal vesicle invasion: validation of Kattan's nomogram in a pathological subgroup. BJU Int 2004;93(7):961964.

4. Ohori M, Scardino PT, Lapin SL, SealeHawkins C, Link J, Wheeler TM. The mechanisms and prognostic significance of semi nal vesicle involvement by prostate cancer. Am J Surg Pathol 1993;17(12):1252-1261.

5. Osborn JR, Ramsden AR, Chodak GW, Persad RA. Should the therapeutic approach to prostate cancer with seminal vesicle invasion be reviewed: improving functional results without diminishing oncological outcome? BJU Int 2004;94(4):482-483.

6. Wang L, Hricak H, Kattan MW, Chen HN, Scardino PT, Kuroiwa K. Prediction of organconfined prostate cancer: incremental value of MR imaging and MR spectroscopic imaging to staging nomograms. Radiology 2006; 238(2):597-603.

7. Bostwick DG, Qian J, Bergstralh E, et al. Prediction of capsular perforation and seminal vesicle invasion in prostate cancer. $\mathrm{J}$ Urol 1996;155(4):1361-1367.

8. Koh H, Kattan MW, Scardino PT, et al. A nomogram to predict seminal vesicle invasion by the extent and location of cancer in systematic biopsy results. J Urol 2003;170(4 Pt 1):1203-1208.

9. Ikonen S, Kärkkäinen P, Kivisaari L, et al. Magnetic resonance imaging of clinically localized prostatic cancer. J Urol 1998;159(3): 915-919.

10. Ikonen S, Kärkkäinen P, Kivisaari L, et al. Endorectal magnetic resonance imaging of prostatic cancer: comparison between fatsuppressed T2-weighted fast spin echo and three-dimensional dual-echo, steady-state sequences. Eur Radiol 2001;11(2):236-241.

11. May F, Treumann T, Dettmar P, Hartung R, Breul J. Limited value of endorectal magnetic resonance imaging and transrectal ultrasonography in the staging of clinically localized prostate cancer. BJU Int 2001; 87(1):66-69.

12. Rørvik J, Halvorsen OJ, Albrektsen G, Ersland L, Daehlin L, Haukaas S. MRI with an endorectal coil for staging of clinically localised prostate cancer prior to radical prostatectomy. Eur Radiol 1999;9(1):29-34.

13. Cornud F, Flam T, Chauveinc L, et al. Extraprostatic spread of clinically localized prostate cancer: factors predictive of pT3 tumor and of positive endorectal MR im- aging examination results. Radiology 2002; 224(1):203-210.

14. Quinn DI, Henshall SM, Brenner PC, et al Prognostic significance of preoperative factors in localized prostate carcinoma treated with radical prostatectomy: importance of percentage of biopsies that contain tumor and the presence of biopsy perineural invasion. Cancer 2003;97(8):1884-1893.

15. Eastham JA, Kattan MW, Riedel E, et al. Variations among individual surgeons in the rate of positive surgical margins in radical prostatectomy specimens. J Urol 2003;170(6 Pt 1): 2292-2295.

16. Zlotta AR, Djavan B, Petein M, Susani M, Marberger M, Schulman CC. Prostate specific antigen density of the transition zone for predicting pathological stage of localized prostate cancer in patients with serum prostate specific antigen less than $10 \mathrm{ng} / \mathrm{ml}$. J Urol 1998;160(6 Pt 1):2089-2095.

17. Perrotti M, Kaufman RP Jr, Jennings TA, et al. Endo-rectal coil magnetic resonance imaging in clinically localized prostate cancer is it accurate? J Urol 1996;156(1):106-109.

18. Presti JC Jr, Hricak H, Narayan PA, Shinohara K, White S, Carroll PR. Local staging of prostatic carcinoma: comparison of transrectal sonography and endorectal MR imaging. AJR Am J Roentgenol 1996;166(1):103-108

19. Bartolozzi C, Menchi I, Lencioni R, et al Local staging of prostate carcinoma with endorectal coil MRI: correlation with wholemount radical prostatectomy specimens. Eur Radiol 1996;6(3):339-345.

20. Wang L, Hricak H, Kattan MW, et al. Prediction of seminal vesicle invasion in prostate cancer: incremental value of adding endorectal MR imaging to the Kattan nomogram. Radiology 2007;242(1):182-188.

21. Wang L, Zhang J, Schwartz LH, et al. Incremental value of multiplanar cross-referencing for prostate cancer staging with endorectal MRI. AJR Am J Roentgenol 2007;188(1):99104.

22. Sala E, Akin O, Moskowitz CS, et al. Endorectal MR imaging in the evaluation of seminal vesicle invasion: diagnostic accuracy and multivariate feature analysis. Radiology 2006;238(3):929-937.

23. Ren J, Huan Y, Wang H, et al. Seminal vesicle invasion in prostate cancer: prediction with combined T2-weighted and diffusion-weighted MR imaging. Eur Radiol 2009;19(10):2481-2486.

24. Kim CK, Choi D, Park BK, Kwon GY, Lim HK. Diffusion-weighted MR imaging for the evaluation of seminal vesicle invasion in 
prostate cancer: initial results. J Magn Reson Imaging 2008;28(4):963-969.

25. Fütterer JJ, Engelbrecht MR, Huisman HJ, et al. Staging prostate cancer with dynamic contrast-enhanced endorectal MR imaging prior to radical prostatectomy: experienced versus less experienced readers. Radiology $2005 ; 237(2): 541-549$.

26. Ogura K, Maekawa S, Okubo K, et al. Dynamic endorectal magnetic resonance imaging for local staging and detection of neurovascular bundle involvement of prostate cancer: correlation with histopathologic results. Urology 2001;57(4):721-726.

27. Hoeks CM, Barentsz JO, Hambrock T, et al. Prostate cancer: multiparametric MR imaging for detection, localization, and staging. Radiology 2011;261(1):46-66.

28. White S, Hricak H, Forstner R, et al. Prostate cancer: effect of postbiopsy hemorrhage on interpretation of MR images. Radiology 1995;195(2):385-390.
29. Schiebler ML, Yankaskas BC, Tempany C, et al. MR imaging in adenocarcinoma of the prostate: interobserver variation and efficacy for determining stage C disease. AJR Am J Roentgenol 1992;158(3):559-562.

30. Hricak H, White S, Vigneron D, et al. Carcinoma of the prostate gland: MR imaging with pelvic phased-array coils versus integrated endorectal-pelvic phased-array coils. Radiology 1994;193(3):703-709.

31. Efron B. Bootstrap methods: another look at the jackknife. Ann Stat 1979;7(1):1-26.

32. Riffenburgh RH. Statistics in medicine. 2nd ed. Amsterdam, the Netherlands: Elsevier Academic Press, 2006; 244, 274.

33. Metz CE, Pan X. "Proper" binormal ROC curves: theory and maximum-likelihood estimation. J Math Psychol 1999;43(1):133.

34. Dorfman DD, Berbaum KS, Metz CE. Receiver operating characteristic rating analysis: generalization to the population of readers and patients with the jackknife method. Invest Radiol 1992;27(9):723-731.

35. Landis JR, Koch GG. The measurement of observer agreement for categorical data. Biometrics 1977;33(1):159-174.

36. Cornud F, Hamida K, Flam T, et al. Endorectal color Doppler sonography and endorectal MR imaging features of nonpalpable prostate cancer: correlation with radical prostatectomy findings. AJR Am J Roentgenol 2000;175(4):1161-1168.

37. Engelbrecht MR, Jager GJ, Laheij RJ, Verbeek AL, van Lier HJ, Barentsz JO. Local staging of prostate cancer using magnetic resonance imaging: a meta-analysis. Eur Radiol 2002;12(9):2294-2302.

38. Berney DM, Wheeler TM, Grignon DJ, et al. International Society of Urological Pathology (ISUP) Consensus Conference on Handling and Staging of Radical Prostatectomy Specimens. Working group 4: seminal vesicles and lymph nodes. Mod Pathol 2011;24(1):39-47. 